\title{
TRANSPARENCIA Y PROTECCIÓN DE DATOS PERSONALES ANTE \\ LOS DERECHOS DE LOS CIUDADANOS EN MÉXICO EN LA ERA DE LA DIGITALIZACIÓN Y EL GOBIERNO ELECTRÓNICO
}

\section{Nancy Nelly González Sanmiguel}

https://orcid.org/0000-0001-9589-2192.Correo: nancygonsa09@hotmail.com

Universidad Autónoma de Nuevo León, México

Doctora en Derecho

\section{Carlos Charles Manzano}

Correo: carlos.charles@ladrilleramecanizada.com

Universidad Autónoma de Nuevo León; México

Doctor en Derecho

\section{Guillermo Cienfuegos Galindo}

Correo: lic.cienfuegos.g.g@hotmail.com

Universidad Autónoma de Nuevo León, México

Doctorante en derecho procesal

\section{RESUMEN}

La inclusión de la utilización de la digitalización para dar esa herramienta hacia la utilización del cambio del gobierno electrónico, ha generado la necesidad de implementar normas para adecuar dichas políticas públicas, señalando en este caso los más importante que es la protección de los datos personales y que una de las características cuando los solicitan es demostrar la finalidad de la utilización de estos, analizando la problemática actual en México acerca de la constitución del Padrón Nacional de Usuarios de Telefonía Móvil externa si cumple con el fin que establece en el decreto que reforma a la Ley Federal de Telecomunicaciones y Radiodifusión.

Palabras claves: Gobierno Electrónico1. ; Reforma Constitucional 2.; Datos Personales 3.; Datos Biométricos 4.; Padrón de usuarios 5.

\section{ABSTRACT}

The inclusion of digitalization in order to provide the tools towards the implementation of an electronic government, has generated the necessity to apply and perform new reference standards or norms to achieve the best execution of these public policies; therefore the most important thing in this case is the protection of personal data, and one of the characteristics when these are requested is to demonstrate its purpose, by analyzing the current problems in Mexico regarding the constitution of the National Register of External Mobile Telephone Users, as well as, questioning if it meets the purpose established in the decree that reforms to the Federal Telecommunications and Broadcasting Law.

Keywords: Electronic Government 1.; Constitutional reform 2.; Personal information 3.; Biometric Data 4.; Register of users 5. 


\section{RESUMO}

A inclusão do uso da digitalização para dar essa ferramenta para o uso da mudança de governo eletrônico, gerou a necessidade de implementar regulamentações para adequar essas políticas públicas, apontando neste caso o mais importante que é a proteção de dados pessoais e aquela das características quando as solicitam é demonstrar a finalidade do uso das mesmas, analisando os problemas atuais no México quanto à constituição do Cadastro Nacional de Usuários Externos de Telefonia Móvel se cumpre a finalidade estabelecida no decreto que reforma o Sistema Federal Lei de Telecomunicações e Radiodifusão.

\section{INTRODUCCIÓN}

En este artículo se analizan los derechos y garantías constitucionales utilizados en el campo de los derechos de la protección de datos con la implicación del cambio de la digitalización del gobierno, siendo por esta conexión una forma en el cómo debe darse la inclusión digital.

Se externarán cuáles son los derechos de los ciudadanos mexicanos y esa libertad referente a nuestros datos personales, que se constituye como una herramienta para poder interactuar con la Administración Pública pero al mismo tiempo, su regulación para la contemplación de un derecho humano que, marcando la paráfrasis de entender la limitación del poder y el cuidado que debemos tener con dicha información.

Examinar los objetivos en México de lograr las expectativas referentes a la conectividad, servicio y la inclusión digital, mismos que vayan acorde con las políticas públicas y en apoyo a los programas para lograr dichas habilidades en los ciudadanos y verificar el avance, marcando así la interoperabilidad y el marco dentro de la normatividad.

Los elementos que constituyen el avance dentro de estos temas será la participación ciudadana en la contemplación de la necesidad de la digitalización y ese cambio hacia la gestión de la Administración Pública para ver las necesidades, estableciendo dentro de las reformas las necesidades que se deben contemplar.

Dentro del capítulo de las reformas Constitucionales se marca cómo se debe ejercer el acceso de la efectividad de la población hacia el gobierno electrónico y se contempla un tema fundamental referente a la protección de los datos personales.

El objetivo de este trabajo de investigación es identificar la relación del cambio del gobierno electrónico y las reformas referentes a este tema con la protección de los datos personales, señalando las garantías que operan en la utilización de este tema, así como señalar los parámetros actuales hacia la operatividad y la funcionalidad de incluir los datos biométricos.

\section{DERECHOS Y GARANTÍAS CONSTITUCIONALES DE LOS MEXICANOS}

El sistema constitucional mexicano reconoce y acredita la relación de la población y sus derechos, así como la distinción que el autor Ovalle Fabela entre los derechos humanos y las garantías, ya que señala que los derechos y humanos tienen consigo una doble fuente, que son la carta magna como se le conoce en el país, así como los tratados internacionales, de los cuales distintos países forman parte 
de esto. Esta perspectiva permite comenzar de forma adecuada el conocimiento sobre el acceso del cual puede gozar el ciudadano mexicano, teniendo en cuenta los derechos pro-persona, universalidad, interdependencia, indivisibilidad y progresividad (OVALLE FAVELA, 2016, p. 149-177).

En estos términos, en un tratado filosófico del saber y entender en cierta manera más concreta y de forma más natural, se le puede conectar con una relación iusfilosófico-política donde se destacan dos formas de fundamentar los derechos humanos y el saber de su entendimiento, así como su comprensión. Esto en referencia sobre las ideologías del gobierno y su gran conexión con la ciudadanía en la forma de transferir información digital que beneficia al usuario.

Pero para lograr un mejor desarrollo de esta investigación, se deben mencionar los principios que el mismo ciudadano cuenta conforme a sus garantías y sus derechos en relación con el acceso digital. Es por tal motivo que se tiene que comprender que los derechos son reconocidos con el simple hecho de estar, ya que pretenden desde un principio el desarrollar un equilibrio y apoyo a la misma población; es por eso por lo que el libre acceso debe ser justo, eficaz, equitativo y coherente. Teniendo en cuenta que el desarrollo de las tecnologías es concebido hace poco y que la misma sociedad ha establecido su pronta evolución. Esto quiere decir que es importante y relevante, ya que como sociedad tenemos una abrupta evolución con las tecnologías. México cada día se va colocando en nuevos estándares, así como un desarrollo muy interesante teniendo en cuenta la relación de nuestros sistemas procesales, así como la justa conexión con el acceso a la justicia.

Dentro del marco condicionado en un estado derecho se debe comprender la existencia de la protección de las garantías y la libertad de los ciudadanos, de los cuales se comprende que en la constitución prevalece la protección de sus derechos naturales. Esto se debe por un proceso objetivo en el que se debe conseguir la igualdad a toda costa, es por tal motivo que subsiste la relación de este equilibrio donde se da un acceso adecuado a sus derechos, así como la libertad de expresión y las decisiones de las cuales se deben manifestar. Por este motivo el derecho durante mucho tiempo cuenta con un desarrollo evolutivo en México, ya que el estado de derecho esta en constante cambio, y el derecho mexicano se encuentra en un estado dúctil de lo cual puede ser muy evolutivo a pasos grandes.

México cuenta con garantías y derechos muy buenos, solo que la difusión de los mismos ha costado mucho trabajo y esto al paso del tiempo no ha enriquecido fehacientemente al ciudadano, por lo cual ha tenido mucho costo, tomando en cuenta lo dicho se debe comprender que la sociedad está en el cambio generacional donde entran las denominadas “TICCAD” que comprenden toda información adecuada y libre para la persona, adecuando organismos que son el sustento o la base importante que genera dicha difusión pero se debe entender que la transmisión de información —como se mencionóha costado cierto trabajo para que la gente pueda analizarla, comprenderla y luego ser utilizada. En México existen diversas plataformas muy importantes de las cuales la ciudadanía puede apoyarse, pero en ciertos casos no la conocen y a la vez puede que no cuenten con herramientas en las que puedan trabajar para conseguir dicho objetivo.

Está claro que México necesita educar de alguna manera con apoyo de las instituciones a la población para que puedan lograr el objetivo que las informaciones o plataformas donde se presentan sus derechos sean transmitidas, solo se necesitan cursos o estrategias que puedan crear una cercanía de la ciudadanía con las plataformas digitales para lograr llevarlos a todos sus derechos y garantías, ya que 
la información existe solo que no se ha prestado la suficiente atención sobre estos temas, mismos que dentro de algunos años cualquiera tendrá ese acceso que ha costado en cierta forma mucho trabajo.

\subsection{Los Derechos como ciudadano en México}

En materia de los derechos del ciudadano, varios autores consideran que existen cuatro condiciones que son básicas como elementos para la participación ciudadana. Estas condiciones consisten en que exista un régimen ya sea democrático, o de otra índole donde se tenga conocimiento de las garantías individuales, así como los canales institucionales, marcos jurídicos y más que nada, la confianza de la ciudadanía hacia los organismos o instituciones democráticas. En relación a esto, son los mecanismos que en un estado o población deben de existir para que verdaderamente logren tener una injerencia adecuada, pero teniendo en cuenta que el mismo ciudadano debe de tener la curiosidad de investigar y saber sobre estos temas, en general ser empáticos a la de realizar una búsqueda o exigencia a las instituciones democráticas (SERRANO RODRIGUEZ, 2015, p. 93-116).

De otra forma lo que se comprende como participación ciudadana es que las autoridades paraestatales y estatales o en general de la república mexicana, adopten decisiones que sean justas y compatibles con la naturaleza de la misma sociedad, así como las normas constitucionales e internacionales donde puedan resultar de manera legitima por haber contado con la justa decisión de los sectores interesados.

Tanto así que tenemos de forma reciente el reconocimiento del derecho humano individual así como el universal a la no discriminación dentro del proceso de un cambio en materia legal, desarrollado para crear una funcionalidad adecuada para ser un instrumento de políticas públicas adecuadas, que este orientado y tenga un sentido en conjunto con el estado, tratando de lograr siempre un mejor desarrollo en cuanto a conocimientos de los cuales puedan disponer de manera sencilla logrando erradicar todo tipo de discriminación entre otros (NIKKEN, 2010, p. 55-140).

La misma ciudadanía esta englobada con un régimen democrático y los derechos que en México se asignan son: de participar, de votar, ser escogido y teniendo en cuenta que pueda ser participe en toda actividad que se desarrolle dentro del país, algunos autores refieren que puede ser un estatus adscriptivo, o más bien atribuido antes de que exista cualquier actividad o acción de manera voluntaria ya que el hecho de pertenecer justifica esa acción. Es por ello que el justo acceso a las distintas plataformas digitales permite un nuevo comienzo en el estado mexicano, ya que es importante para los ciudadanos tener esa justa participación ciudadana (FRANCO CUERVO, 2018, p. 189-208).

El ser humano por naturaleza es libre, comprendiendo con esto que toda decisión que sea tomada debe ser libre y justa, pero siempre en cada sociedad a lo largo de los años se ha ido modificando mediante prueba y error, lo que conlleva siempre una progresiva evolución y comprensión de las cosas.

En México se tiene una estadística proporcionada por el Sistema Nacional de Información Estadística y Geográfica, estos datos arrojados por la encuesta Nacional sobre Disponibilidad y Uso de Tecnologías de la Información en los Hogares de 2019, el 70.1\% de la población de seis años o más en México es usuaria de Internet.

- 20.1 millones de hogares (56.4\% del total nacional) disponen de conexión a Internet. 
- De la población con estudios universitarios, el $96.4 \%$ se conecta a la red, mientras que del grupo de personas con estudios de educación básica se conecta el 59.1\%.

Estas cifras fungen como herramientas que se pueden utilizar para la protección, atención y servicio al ciudadano. En el caso de México esto se ha vuelto claro en años recientes, pues de acuerdo con los datos que la encuesta Nacional sobre Disponibilidad de Tecnologías de Información en Hogares del Instituto Nacional de Estadística y Geografía (INEGI, 2016a) ofrece, más de la mitad de los hogares en el país no disponen de conexión a internet (53\%); de 32,925,270 hogares solo 45.6\% cuentan con computadora y de $122,273,473$ habitantes $73.6 \%$ son usuarios de telefonía móvil. Las cifras anteriores reflejan que existen grupos de personas con un rezago en el acceso y uso de estas tecnologias (ESTADISTICAS... 2021).

Para colocarnos en un contexto mejor, los derechos de los ciudadanos siempre se han establecidos, pero estos a lo largo de los años conforme la sociedad evoluciona ellos también lo hacen, por tal motivo en México se han desarrollado plataformas, las cuales colocan la información a primera mano, esto con el sentido de proteger al ciudadano con su libertad de conocer todos sus derechos. Sin embargo, esto ha costado trabajo realizarlo, ya que en México el promedio de conocimiento de las tecnologías era bajo, y según algunos autores, el crecimiento de ideas sobre las nuevas tecnologías va en aumento, sin que esto quiera decir que lo sea en realidad, teniendo en cuenta que hace un corto tiempo se ha forzado al usuario a lograr entenderse con las tecnologías creando en ellas una unión muy importante.

Para llevar un paso más, se define a las TICCAD como las herramientas y procesos para lograr acceder, guardar, organizar, producir, intercambiar y presentar información de forma electrónica, teniendo como antecedente que las tecnologías comenzaron a difundirse a partir de los años setenta y ochenta, siendo preciso e importante mencionarlo, pues se observa que va en aumento el uso de estas plataformas, aunque se debe seguir promocionando, ya que falta un largo y arduo caminho (MARTÍNEZ DOMINGUEZ, 2018).

En México acuñamos el término de sociedad de la información, con el cual se establece que somos sociedades modernas y de gran avance, de lo cual la información ha sido de gran riqueza, siendo de gran apoyo, dado que debe existir la promoción del conocimiento para tener una mejor comunicación y entendimiento.

Esto se deriva de un impulso que 90 países desarrollados promovieron a través de la liberación de las telecomunicaciones, para que se pudiese generar un nuevo modelo de tecnología y la comunicación. Esto se llevó a cabo dentro de la cumbre mundial de la sociedad de la información, todo esto tomo unos años para que se llevara de forma adecuada, pues se buscaron soluciones y el establecimiento de un acuerdo en los campos de gobierno de internet, siendo esto parte importante, pues se debe tomar en cuenta que México tiene una gran participación (LA CUMBRE MUNDIAL...2021).

Todo este proceso en México cuesta mucho trabajo, porque se debe crear una campaña de concientización de la era de las nuevas tecnologías, la cual lleva muy poco tiempo en el mercado mexicano, ya que en otros lugares se ha desarrollado en una manera exponencial.

A lo que a razón de los derechos de los ciudadanos se debe establecer, es que no se cuenta con cierta cobertura de manera correcta o que tal vez la cultura del país no coadyuva de forma adecuada, teniendo en cuenta que México tiene un estado de derecho reconocible, en el que la conformación de 
sus leyes locales y federales son establecidas con buen criterio, así como su carta magna, esto comprende que el sistema jurídico es riguroso, solo se necesita lograr transmitir la información de forma adecuada y entendible.

Aunado a esto se debe contemplar el significado más apropiado para generar un concepto que lleve a cabo la relación con el libre acceso y la transferencia de documentos, entre otros, se le puede conocer como la forma de transmisión de datos del ciudadano pero para ser más relevante se debe comprender que debe existir el acceso sobre ese sistema, la forma de la que obtiene la información, la búsqueda de atraer al usuario al utilizar dichas herramientas de manera que sea de su interés, dado que esto repercute por su situación en la que se encuentre y tratar que sea de manera sencilla (MARTÍNEZ; DE MINGO, 2018, p. 6-16).

\subsection{La información en México sobre un gobierno electrónico}

En México se cuenta con plataformas adecuadas que conllevan la buena relación con la ciudadanía y el estado, se menciona que se encuentran estables y que la información es precisa y oportuna, donde se describe toda la información importante. Pero otra de las situaciones que suceden es que existe, pero no es utilizada, y se encuentran en ciertos casos obsoletas, de aquí nacen ciertas preguntas, porque se cuenta con información de primera mano y nadie las utiliza, ¿será que las mismas plataformas no son difundidas o en cierta forma se debe ser profesional del área social como abogados en su caso para que conozcan sobre esos micrositios o por la situación que la ciudadanía no está interesada en ellas?

El resultado es que la ciudadanía no busca información, sino que la localiza cuando necesita o depende de ella para realizar alguna operación, esta información se debe buscar, ser promovida, pero ello no se promueve por una parte los gobiernos que no apoyan como se merece a los ciudadanos, ya que también la mayoría de la población no cuenta con recursos para contar con una herramienta que permita utilizarlo y además no se le enseña cómo se debe usar, pero esto de cierta forma se comprende por culpa de la política que se realiza en México, así como la economía y demás variantes que suceden en el país.

En estos países se debe tener en cuenta que a veces es difícil realizar un salto tan abrupto, pero se debe tomar en cuenta realizarlo, y para ello se debe considerar que se ocupa mayor participación ciudadana, así como el alcance de tecnología en plazas públicas, porque se considera que las nuevas tecnologías como lo es el internet, deberían ser gratuitas ya que es algo que comprende la libertad de comunicación y que los gobiernos deben darse cuenta que en ellos recae la responsabilidad de lograr ese enlace de contar con internet para todos y no solo difundirlo, sino además enseñarlo a la población.

Es necesario concientizar a la población para que los gobiernos apoyen para alcanzar un libre y buen desarrollo de las actividades tecnológicas que en verdad se necesitan, pues la trascendencia de un estado se debe al trabajo en equipo, así como el apoyo de los organismos para estatales, centralizados y descentralizados entre otros. México cuenta con mucho potencial que no ha sido proyectado o más bien explotado como se debe.

Esto describe un panorama muy particular sobre las políticas de acceso y también el uso de las nuevas tecnologías en México, teniendo un parámetro previsto que tome como referencia las TICCAD como sistema o mecanismo auxiliar. Algunos autores mencionan sobre la creación del sistema nacio- 
nal e-México como una agencia coordinadora de los diferentes organismos gubernamentales con el propósito de poder lograr un impulso tecnológico y que pueda de esta manera crear un beneficio a la misma población (AVILA BARRIOS, 2014, p. 263-288).

El sistema e-México cuenta con tres objetivos que son conectividad, servicios digitales y contenido, además de la inclusión social, creando así la agencia digital en México, después de un cierto tiempo se creó la estrategia digital nacional como plan para fomentar la adopción de mecanismos y herramientas, que insertaran la información del conocimiento basándose en ciertos objetivos de transformación gubernamental, economía digital, educación de calidad, seguridad ciudadana, entre otros. Estos objetivos deben de ser aplicados por y a los gobiernos, sobre todo en los cuales ofrecen dicho acceso a la información, requiriendo de las siguientes condiciones necesarias para alcanzar las metas de la estrategia: conectividad; inclusión; habilidades digitales; interoperabilidad; marco jurídico, y datos abiertos (NAVARRO, 2018, p. 49-64).

\subsection{Transparencia del gobierno electrónico}

Dentro de la república mexicana el derecho al acceso a la información siempre se ha establecido, aunque no se tenía un parámetro como tal en el pasado, y era algo normal ya que no se contaba con la herramienta adecuada que hoy en día se tiene. Dentro de esto la información debe ser siempre libre para los usuarios, pero estos dependen de cómo las administraciones influyen y actúan con buenos mecanismos que conlleven la causa de transferir información de buena forma, para ello se tiene que tomar en cuenta toda la estadística que se ha desarrollado a lo largo de la década que menciona el porcentaje y la forma de comunicación con la ciudadanía. creando una relación estrecha entre el estado y las personas. A esto se le denomina como las nuevas tecnologías de las cuales en un futuro no muy lejano dejarán de ser poco utilizadas a ser muy utilizadas.

Esto demuestra que la transparencia en sus formas diferentes logra ser cada vez más importante para lograr fortalecer la democracia y esto también ofrecer a la ciudadanía más posibilidades de poder tener control y ser más participes. Logrando como objetivo convertirse en una fuente importante de progreso para ser comparativo de países en proceso de democratización.

De esto se desprenden otras herramientas que se encuentran vinculadas, es decir, el gobierno electrónico que contiene ciertas promesas de tratar de hacer más eficiente al gobierno y también de ahorrar recursos, donde se proveerá a la población la información que verdaderamente necesita para poder evaluar, dándoles más participación así como la libre elección de datos que sean más útiles, como participar en procesos políticos. Está claro que no cuesta soñar, pero esto es un determinante que se va a tardar en cierta forma para que las disparidades tecnológicas no tengan participación directa o ya sea asunto de privacidade (HAVRILUX; SALAZAR, 2015, p. 345-361).

Se menciona que a nivel internacional se ha progresado notablemente, teniendo en cuenta que son resultados diferentes, no obstante nos hace pensar como mexicanos que pronto tendremos la misma cercanía con la utilización de bases de datos y acceso a una justa transparencia donde el actuar del gobierno electrónico sea válido y justo. 
A lo que refiere la transparencia de la información pública se obligada a abatir la corrupción donde logre optimizar la eficiencia en cuanto procesos o desarrollos de algún sistema y tener la máxima posibilidad de mantener una paz donde lograran nutrir con contenido que realmente valga la pena.

Por otro lado, mencionan algunos autores que la transparencia se divide en dos grupos de ejecución de política de acceso a la información. Por una parte, existen políticas de primera generación, que asientan a abrir los archivos oficiales o de acceso a la información pública y son conocidas como el acceso a la información. Dichas políticas discuten la propuesta de forma vertical, que mantiene que, si se cuenta con acceso a la información pública, los ciudadanos podrán contar con herramientas fundamentales para lograr controlar de forma eficiente lo que hacen los gobiernos, así como fortalecer la participación ciudadana y de la nueva democracia tecnológica (PINHO DE OLIVEIRA, 2020, p. 63-88).

En otro sentido, el de segunda generación que se denomina como transparencia focalizada, tiene el objetivo de divulgar la información específica, identificando y diagnosticando un objetivo, teniendo en cuenta que dicha información establecida al observarla puede crear un resultado desinformado y no adecuado como tal, por lo que esto debe ir acompañado de capacitaciones y promociones públicas.

\subsection{Acceso a la información pública}

El acceso a la información se ha suscitado durante mucho tiempo atrás. Esta información es conducida por elementos creados por la misma sociedad con el desarrollo de su entorno y su evolución, como en los tiempos de Aristóteles donde ha destacado la importancia de la evolución de la democracia donde los ciudadanos tienen el libre acceso a la información y que pueden juzgar y determinar ciertos criterios que tienen el objetivo principal de lograr un equilibrio como nación. Se debe tener en cuenta que la información es algo muy importante y debe ser transmitida, sin embargo se tiene en cuenta que un uso malo de la información es utilizado hoy en día como constante práctica.

Es por tal motivo que el acceso a la información pública se localiza de forma orientada a la transparencia y a la participación ciudadana. Se concede la relación de forma espontánea, se comprende que la información trabaja en función de la participación ciudadana, ya que estos mismos no generan impacto sobre fenómenos, solo se sustenta tratar de otorgar un acceso justo y que el gobierno sea ético y responsable.

La visión que versa sobre la transparencia, es que esta sea un instrumento realmente de gran utilidad, así como las condiciones con las que se presenta dicha información, teniendo en cuenta que los objetivos sean válidos, directos y reales. A esto se debe sumar el trabajo que los gobiernos desarrollan, permitiendo trabajar en conjunto la estrategia digital y el gobierno, con esto se busca condicionar el llevarlos a un camino digital para lograr la transmisión de la información.

México va en constante evolución, teniendo en cuenta toda política que se contraviene y esto conlleva una justificación de cada gobierno en modificar, sin llegar a los mismos condicionamientos, convirtiéndose en ciclos operativos que no determinan un buen avance, ya que dependen de la situación gubernamental para que esto se pueda llevar a cabo, pero este acceso a la información pública cuenta con bases de datos de los cuales el gobierno sustrae y analiza, teniendo en cuenta que no siempre satisface como en realidad se debería hacer, pero es aquí donde la participación del ciudadano tiene un papel muy importante para colaborar e impulsar políticas públicas estables y bien desarrolladas. 


\subsection{Participación ciudadana}

La participación nace de un colectivo de la sociedad o de masas que conviven día a día unos con otros, pero esta participación no aparece de manera mágica en un régimen como lo es el democrático, ya que en cierta forma es determinada por ciertas condiciones, tomando en cuenta las garantías individuales, mismas que permiten al hombre y al estado convivir de manera adecuada, logrando un equilibrio importante. También los canales institucionales y los marcos jurídicos determinan la columna vertebral de la ciudadanía, es por ello que se debe contar con información eficiente, clara y concisa para lograr ese objetivo y como elemento final la confianza de la ciudadanía hacia los organismos institucionales, ya que eso es lo más importante reconocer.

En un contexto general, la participación de los ciudadanos es importante para moderar y manejar el poder político, ya que pretende ser funcional a la toma de decisiones. A esto se hace la distinción que lo anterior no se limita en el simple hecho de un voto tomándolo como ejemplo, sino el caso es que la participación ciudadana sea motivada para más cosas como lo es la participación social, comunitaria, política y ciudadana. Existen varios conceptos diferentes de lo que entrañan, la participación social se entiende como el hecho de encontrarse activo en una asociación u organización, grupo de personas que colabora en conjunto para un fin común.

Otra de las participaciones es la comunitaria, la cual tiene como objetivo que los individuos logren reunirse para lograr un mayor bienestar, buscando el desarrollo de la misma sociedad. Otra de las columnas importantes es la participación política, la cual tiene mucha importancia, ya que con ella se escoge al gobierno que brindará un acceso digital, así como la justa toma de decisiones en colaboración con la participación ciudadana, y como definición final de la participación ciudadana se puede decir que es la relación que tiene la ciudadanía con el estado para lograr un buen desarrollo en la administración pública.

Este desarrollo es muy importante ya que nos permite vigilar, controlar la gestión de los gobernantes y además se hace escuchar y puede ser participe en la toma de decisiones, esto teniendo en cuenta los cambios evolutivos de la misma sociedad que en su entorno se establecen.

\section{REFORMA CONSTITUCIONAL TELECOMUNICACIONES}

Con respecto a la garantía que está ofreciendo el cambio de la digitalización y la transición jurídica de la Administración Pública y los derechos de los ciudadanos al proceso del cambio de los modelos sociales y en raíz de la reforma regulatoria dentro del sector de la Telecomunicación, se ha incorporado la globalización y la entrada en vigor del Tratado de Libre Comercio del Norte, dando esto un impacto en las reformas actuales de la digitalización y siendo reconocidos como derechos fundamentales al momento de ser positivizados dentro de la constitución. Contemplando aquí la necesidad de la nueva era en el acceso a la tecnología de la información y comunicación, consagrada dentro de los artículos 6 y 7 constitucionales, dando enfoque de servicios públicos a la utilización del internet y la banda ancha, por tal motivo se exige que sea de interés general la competencia, calidad, cobertura universal, la operatividad de estos servicios, así como tener una estructura adecuada para la difusión y la conectividad. 
El objetivo de este trabajo consiste analizar estos derechos y garantías que tenemos en referencia a las telecomunicaciones, las condiciones con respecto a la conectividad, competencia, cobertura universal, así como los instrumentos gubernamentales que se utilizan para dar esta regulación con respecto a las metas que se deben contemplar para asegurar la integración de la sociedad dentro de la inclusión tecnológica con respeto a los derechos.

Esta gestión de gobierno señala 5 puntos importantes para el apoyo de la inclusión digital en México mencionado que son:

"Impulso a la cobertura social y acceso asequible; Desarrollo de habilidades y capacidades digitales; Identificación de tecnologías, protección a la integridad del dato, posibilidades de interoperabilidad e impulso a la ciberseguridad, como partes fundamentales del desarrollo tecnológico; Evaluación y participación interinstitucional para generar políticas públicas acordes con el desarrollo y convergencia de las nuevas tecnologías; y Promoción de infraestructura de telecomunicaciones y radiodifusión" (IMPLEMENTA...2021).

Con esto se busca lograr un impulso en nuestro país para tener una cobertura social enfocada hacia la población indígena y marginada, para ir cerrando bloques con respecto a la brecha digital e incrementar la confianza en la utilización de medios electrónicos, esto se consideraba antes de la pandemia dentro del proyecto denominado "Marco de Habilidades Digitales", refiriendo el punto de partida que se entiende por una habilidad digital y clasificándola para poder llegar al objetivo de la inclusión digital.

Esto genera un marco de habilidades digitales, puesto que tales programas procuran dar esa capacitación para que la ciudadanía torne hacia la digitalización y conozca los derechos que tienen, estableciendo las estrategias y acciones que se van a implementar dentro de los proyectos, política y programas dentro de los sectores de la Administración Pública. Dando cumplimiento a lo establecido en la Declaración Universal de los Derechos Humanos referente al artículo 19 que establece: “Todo individuo tiene derecho a la libertad de opinión y de expresión; este derecho incluye el de no ser molestado a causa de sus opiniones, el de investigar y recibir informaciones y opiniones, y el de difundirlas, sin limitación de fronteras, por cualquier medio de expresión” (DECLARACIÓN...2021).

La sociedad tiene este derecho de comunicarse a través de las redes sociales y se ha convertido este acceso a la tecnología en un poder para la modificación de las políticas públicas, comprendiendo que no solo se trata de un derecho de libertad de expresión sino que se consideran "como espacios de poder y que, al mismo tiempo, amplían el sentido de defensa de la libertad de expresión en los campos de disputa por los significados de inclusión digital" (RIBEIRO ROSA, 2021). Es la importancia de determinar esta inclusión de los ciudadanos y poder dar un estatus dentro de la digitalización y los derechos que consagran dentro de la Administración Pública.

La inclusión digital es un derecho y una garantía de los ciudadanos con respecto al desarrollo tecnológico, desde el aspecto social y económico estos nuevos derechos sociales están dentro del desafío de la configuración de las herramientas digitales dentro de la normatividad jurídica, es por tanto la importancia del estudio de las garantías y los derechos que tenemos como ciudadanos dentro de esta nueva era y el cambio de las políticas públicas hacia inclusión digital. 


\subsection{Acceso efectivo de la población}

Previendo la garantía de un acceso a la información y comunicación por medio de la tecnología, el Ejecutivo Federal sienta las bases para establecer la accesibilidad y la conectividad dando paso a establecer una red compartida por medio de la Comisión Federal de Electricidad, utilizando en la banda de $700 \mathrm{MHz}$ que se utiliza para la transición a la revisión digital terrestre que se remite a través del espectro liberado de al menos $90 \mathrm{MHz}$, generando con esto los recursos de una red troncal de fibra óptica, tratando por medio de programas del Plan Nacional de Desarrollo, la generación de espacios públicos donde alcance la cobertura universal.

La acciones y programas que se presentan dentro de la Secretaría de Comunicaciones y Transportes desarrollan el programa de Conectividad en Sitios Públicos, constituyendo esto la finalidad de dar la cobertura universal y viendo esto una herramienta de proporcionar la oportunidad de ingresar a la digitalización y enfocarse en una política pública, dando además los servicios que son necesarios para la transformación del gobierno electrónico para considerar los elementos que son la banda ancha e internet para dar conectividad con los sitios que deben ser contemplados, estableciendo el Programa cuáles son los sitios públicos que deben conectar y dar la conectividad efectiva con la inclusión digital para llevar el cambio dentro del gobierno, es por eso la necesidad desarrollar tales medidas para llegar al objetivo.

\subsection{Protección de Datos}

La digitalización y el paso al gobierno electrónico y la obtención de datos a través de los procesos digitales da comienzo hacia la protección de la información que se obtiene por parte del gobierno y de la sistematización dentro de los procesos que solicitan varios datos dentro de los trámites, derivando este tema en el resguardo de la información que se obtiene a través de estos y cómo puede causar una vulnerabilidad la exposición de estos.

Siendo las dos caras de la moneda la tecnología, la facilitación de la información y a la vez la oportunidad de obtener la misma de una manera rápida y efectiva, se empieza a determinar cómo se debe ejercer la garantía hacia la protección de los datos personales, concibiendo la flexibilidad de la exposición de estos a través de la tecnología, forjando por medio de los instrumentos legislativo dar esa seguridad, siendo así que este tratamiento de los datos personales se ejerce por medio de un control para utilizar los datos proporcionados.

La globalización ha externado la importancia de la injerencia dentro de los datos personales y cómo se pueden exportar y proporcionar a raíz de la implementación de las redes sociales y el internet, dando con esto el paso de una barrera territorial de la expansión de la información, por eso la importancia de tener el control por parte de la Administración Pública, la manera de solicitarlo y el resguardo de esta información que constituye la cúspide del cambio hacia la manera de interactuar y a través de una normatividad que contemple las reglas que deben tener los sujetos, es la forma en el cómo podemos tener una limitación en la exposición de dicha información.

En México esta forma de adquirir datos de los ciudadanos se ha estado aplicando, cada vez se ha implicado y desarrollado más la utilización de la tecnología para la obtención de los datos personales, 
pero ahora se trata de inducir que, por medio de un padrón se puede generar una base de elementos de datos biométricos, en particular en el año 2021 se está generando por medio de un padrón de quienes utilizan las líneas telefónicas, generando polémica en torno a la seguridad que el Estado tiene que brindar en la protección de los datos personales, la razón para pedir este tipo de padrón y el tema relacionado con el robo identidad, con esto se pretende tener una comprobación de la identidad de la persona que cuenta con la línea telefónica.

Empezamos por analizar que dentro de la normatividad no se define qué se entiende por datos biométricos. Esta definición es proporcionada por el Instituto Nacional de Transparencia, Acceso a la Información y Protección de Datos Personales (INAI) que establece lo siguiente: "son las propiedades físicas, fisiológicas, de comportamiento o rasgos de la personalidad, atribuibles a una sola persona y que son medibles" (GUIA...2021). De esta manera se podrán tener características particulares de cada persona, la huella digital, reconocimiento facial, el iris, pulsación cardíaca, entre otras de cada persona.

Para garantizar el derecho hacia la protección de los datos personales se deben considerar los principios, los deberes y los derechos que deben tener los titulares de ellos para considerar los aspectos de un cumplimiento de resguardar dicha información.

En lo que respecta a las modificaciones con la aprobación de la utilización de datos biométricos dentro del Padrón Nacional de Usuarios de Telefonía Móvil y a través del DECRETO publicado el 16 de abril de 2021, que contempla las reformas y adiciones a la Ley Federal de Telecomunicaciones y Radiodifusión, dicho padrón es creado para dar efectividad a la información por medio de la base de datos proporcionados por las compañías telefónicas y la exigencia es para todo aquel que tenga un Plan Técnico Fundamental de Numeración con el propósito de dar seguridad y justicia en la relación con la comisión de delitos.

Esto genera situaciones a partir de la intención de solucionar un problema, estableciendo métodos que no tienen una manera confiable de proporcionar la seguridad de la obtención de los datos, y en el segundo lugar utilizar terceras personas, es decir a las compañías telefónicas, mismas que tienen la concesión de ese servicio para realizar este procedimiento, para después pasar ese padrón al Instituto Federal de Telecomunicaciones, generando aquí un traspaso de información. Dentro de dicho decreto señala que se validará la información triangulando la información, así como también se buscará una seguridad dentro de los datos que se proporcionen a través de los datos biométricos, considerando las infracciones hacia las concesionarias en caso de que incurran en una anomalía dentro del proceso de la captura de los datos biométricos de las líneas telefónicas.

Pero aquí empezamos analizando la Ley Federal de Protección de Datos Personales en Posesión de los Particulares, la cual señala en el artículo 1 que: "tiene por objeto la protección de los datos personales en posesión de los particulares, con la finalidad de regular su tratamiento legítimo, controlado e informado, a efecto de garantizar la privacidad y el derecho a la autodeterminación informativa de las personas" (ESTADOS UNIDOS MEXICANOS, 2010). Y manifestado que los datos personales son: "Cualquier información concerniente a una persona física identificada o identificable", de manera que los datos biométricos son considerados, pero dentro de esta normatividad existe una limitación, mencionado la finalidad y que la recolección de datos será por un tercero indica la necesidad de tomar 
las medidas pertinentes para recabar dicha información, señalando también la utilización del aviso de privacidad que será como un candado de seguridad dentro de esa relación jurídica.

Pero ahora, dentro de la modificación a través del decreto antes señalado no se menciona cuáles serán las seguridades que el Instituto brindará para tal efecto, sí señala la operación, las obligaciones y deberes de la concesionarias, así como las infracciones al momento de no cumplir con los lineamientos, pero solo menciona que al momento de utilizar los medios digitales así como la utilización de medios remotos, se emitirán lineamientos para garantizar la veracidad de la información dentro de dichos padrones, pero no establece que dicha información no será utilizada por alguien más o que podrán vender dicho padrón o filtrar información, conteniendo aún más datos que nos relacionan físicamente con nuestras características e identificación personal, sin considerar las consecuencia que puede provocar el solicitar dicha información.

Siendo así que esto violenta un derecho humano debido a la utilización de dichos datos biométricos no cumple con la funcionalidad de impedir que siga la delincuencia, es decir, esto no es la manera de evitar los ilícitos dentro de un país, además que la información que se arroja dentro de esta base de datos personales son características fisiológicas o rasgos conductuales, de manera que el daño que se puede provocar para evitar un supuesto delito puede ser mayor, por lo que no se cumple ni con el principio de la finalidad, ni proporcionalidad al solicitar dichos datos biométricos.

El principio de proporcionalidad solicita que el aviso de privacidad vaya acorde a los elementos del por qué se solicitaron dicho datos, es decir que la relación de la finalidad para solicitarlo es el motivo por el que se proporcione, viendo aquí quien solicita dicha deben ser los concesionarios de las telefónicas y no es para su control, sino que esta información se pasará a la institución para tener dicho padrón, y evitar un posible ilícito, sin considerar que en nuestra realidad se están dando más elementos para realizar más ilícitos en nuestro país y perjudicado a los usuarios de las telefonías y a los concesionarios, debido que su finalidad no es perseguir los ilícitos ni realizar padrones para evitarlos, esa es una función jurisdiccional que le pertenece al Estado, de manera que están excediendo dichas facultades.

Es por eso, por lo que dentro de este principio no resulta necesaria la obtención de dichos datos con la finalidad que se busca, siendo que no cumple de acuerdo con el artículo 13 de Ley Federal de Protección de datos Personales en Posesión de los Particulares, mismo que a letra dice: "El tratamiento de datos personales será el que resulte necesario, adecuado y relevante en relación con las finalidades previstas en el aviso de privacidad. En particular para datos personales sensibles, el responsable deberá realizar esfuerzos razonables para limitar el periodo de tratamiento de los mismos a efecto de que sea el mínimo indispensable" (ESTADOS UNIDOS MEXICANOS, 2010). Es así como no existe una relación entre la finalidad para la utilización de dicho padrón.

Con lo que respecta al principio de la finalidad se abarca que dentro del artículo 12 de la ley antes señalada, y menciona lo siguiente: "Si el responsable pretende tratar los datos para un fin distinto que no resulte compatible o análogo a los fines establecidos en aviso de privacidad, se requerirá obtener nuevamente el consentimiento del titular" (ESTADOS UNIDOS MEXICANOS, 2010). Como se demuestra lo referente a los datos biométricos que se solicitan dentro de este padrón, su objetivo no va acorde para la utilización del servicio telefónico, desde nuestro punto de vista no cumple el objeto para la captación de dicha información, no siendo objetiva la utilización de dicho padrón. 
Por tales motivos consideramos que existe una violación hacia la privacidad de nuestros datos personales al no establecer una forma de seguridad para la utilización de estos, la manera cómo plantean y el objetivo para la realización de este padrón es una manera de violentar nuestros derechos y en lugar de combatir un problema estarían acarreando un problema mayor, debido a que no se establecen las formas de seguridad electrónica que deberían tener para dichos padrones.

\section{CONCLUSIÓN}

La reforma constitucional referente a la Tecnología se ha dirigido en un marco hacia la globalización, ejerciendo una manera de exigir el respeto de los ciudadanos desde la perspectiva de la facilidad para la conectividad, accesibilidad, concibiendo dentro de la Constitución esa protección hacia los datos personales, otorgando el derecho, acceso, rectificación como oponibilidad de dichos datos personales.

Por medio de la normatividad jurídica y con la intención de vigilar y proteger la seguridad de los ciudadanos, se debe destacar la importancia que tiene proporcionar estos medios de identificación de las personas y cuál es la finalidad y el objetivo para solicitar dicha información, por lo tanto se debe constar el hecho de lo que están solicitando vaya acorde a las necesidades por lo que solicitan esa información.

Aquí vemos un primer punto dentro de este cambio hacia la digitalización y la utilización de los datos personales para poder acceder al gobierno electrónico, sin considerar que debemos respetar la información que sea proporcionada al igual que es necesario establecer medidas para constar la efectividad de la Administración Pública y que no vaya suceder un hackeo o una fraude digital con dicha información que se proporciona de manera voluntaria por los sujetos que proporcionan los datos que se requieren.

Se contempla dentro de la norma jurídica la libertad que tenemos de externar nuestra opinión en cualquier medio de comunicación, esto implica las redes tecnológicas. Este cambio ha exigido que el Estado ofrezca de manera correcta la forma de cómo interactuar de manera digital, dando así el crecimiento dentro de la tecnología y la sociedad para desarrollar los nuevos derechos sociales consistentes en dar un acceso efectivo al gobierno electrónico.

Referente al tema de la protección de datos en México, estamos viviendo una etapa en la que se están exigiendo a través de un decreto la utilización de ciertos datos biométricos para generar un padrón de los usuarios con el objetivo de utilizarla en caso de que con ese número se cometa algún ilícito ejerciendo así de manera incorrecta esta justificación.

Contar con registro de los datos biométricos de los ciudadanos que cuentan con una línea telefónica, es un riesgo fuerte, pues estos se pueden considerar como parte fundamental de una persona, sobre todo porque no se establece la forma en cómo va a proteger dicho padrón los datos y los lineamientos de la comunicación, ya que quienes lo realizarán serán las concesionarias, mismas que tendrán que dar oportunidad al Instituto Federal de Telecomunicación de su resguardo y utilización de dicha información, aunque este no sería el encargado de utilizar dicha información, ya que sería el poder judicial, quien en caso de la comisión de algún ilícito requeriría dicha información. Esta situación constituye una gran complejidad, pues muchos sujetos serían intermediarios hasta alcanzar el fin para el cual fue creado.

Siendo también importante comprender cuál o cuáles serían las sanciones correspondientes por hacer un mal uso de la información que usurparon de dicho padrón, en estos casos qué es lo que proce- 
dería, pues se está hablando de rasgos característicos y únicos de cada persona, como lo pueden ser las huellas digitales. En conclusión podemos señalar que esta cuestión que estamos presentando en México representa un exceso al exigir y no proteger los datos biométricos de los ciudadanos, y esto puede derivar en una gran cantidad de amparos, pues se deben proteger los derechos que la constitución reconoce.

\section{BIBLIOGRAFÍA}

ÁVILA BARRIOS, Delia, "El uso de las TICs en el entorno de la nueva gestión pública mexicana”, en Andamios, 2014, vol. 11, no 24, pp. 263-288.

ESTADOS UNIDOS MEXICANOS. Ley Federal de Protección de Datos Personales. México, 2010. Véase en: http://www.diputados.gob.mx/LeyesBiblio/pdf/LFPDPPP.pd

FRANCO CUERVO, Juan José, "Los derechos político-electorales de los ciudadanos en México a la sombra de una democracia y una tutela delegativas", en Revista IUS, 2018, vol. 12, no 42, pp. 189-208. HAVRILUK, Lubiza Osio; SALAZAR, Pedro Luis Pineda, "Propuesta metodológica para evaluar el gobierno electrónico en las universidades autónomas venezolanas", en Quórum Académico, 2015, vol. 12, no 2, pp. 345-361.

MARTÍNEZ, Agustín Cerrillo; DE MINGO, Anahí Casadesús, “El impacto de la gestión documental en la transparencia de las Administraciones públicas: la transparencia por diseño”, en Gestión y Análisis de Políticas Públicas, 2018, pp. 6-16.

MARTÍNEZ DOMÍNGUEZ, Marlene, "Acceso y uso de tecnologías de la información y comunicación en México: factores determinantes", en PAAKAT: revista de tecnología y sociedad, 2018, vol. 8, no 14.

NAVARRO, Dulce Angélica Gómez, et al. "La brecha digital una revisión conceptual y aportaciones metodológicas para su estudio en México", en Entre ciencias: diálogos en la sociedad del conocimiento, 2018, vol. 6, no 16, pp. 49-64.

NIKKEN, Pedro, "La protección de los derechos humanos: haciendo efectiva la progresividad de los derechos económicos, sociales y culturales”, en Revista Iidh, 2010, vol. 52, no 1, pp. 55-140.

OVALLE FAVELA, José, “Derechos humanos y garantías constitucionales”, en Boletín Mexicano de Derecho Comparado, vol. XLIX, núm. 146, Universidad Nacional Autónoma de México, Distrito Federal, México, mayo-agosto, 2016, pp.149-177.

PINHO DE OLIVEIRA, María Fátima, "Gobierno Abierto y Participación Ciudadana. Algunas normas necesarias en Venezuela", en Derecho global. Estudios sobre derecho y justicia, 2020, vol. 5, no 15, pp. 63-88.

RIBERIRO ROSA, Fernanda, "Inclusión Digital como políticas públicas: disputas en el campo de los derechos humanos". Véase en: https://www.corteidh.or.cr/tablas/r32486-1.pdf

SERRANO RODRÍGUEZ, Azucena, “La participación ciudadana en México”, en Estudios políticos (México), 2015, no 34, pp. 93-116. 


\section{Documentos sitios web}

Declaración Universal de Derechos Humanos Véase en: https://www.un.org/es/about-us/ universal-declaration-of-human-rights

Estadísticas a propósito del día mundial del internet. Véase en: https://www.inegi.org.mx/contenidos/saladeprensa/aproposito/2020/eap_internet20.pdf

Guía para el tratamiento de Datos Biométricos. Véase en: https://home.inai.org.mx/wp-content/documentos/ DocumentosSectorPrivado/GuiaDatosBiometricos_Web_Links.pdf

Implementa SCT cinco ejes estratégicos para apoyar la inclusión digital de México: SJV. Véase en: https://www. gob.mx/sct/prensa/implementa-sct-cinco-ejes-estrategicos-para-apoyar-la-inclusion-digital-de-mexico-sjv La Cumbre Mundial sobre la Sociedad de la Información y la brecha de la banda ancha: obstáculos y soluciones. Véase en: https://www.un.org/es/chronicle/article/ la-cumbre-mundial-sobre-la-sociedad-de-la-informacion-yla-brecha-de-la-banda-ancha-obstaculos-y 